\title{
Enhancing Traditional Behavioral Parent Training for Single Mothers of Children with ADHD
}

\author{
Anil Chacko, Brian T. Wymbs, Frances A. Wymbs, William E. Pelham, \\ Michelle S. Swanger-Gagne, Erin Girio, Lauma Pirvics, Laura Herbst, \\ Jamie Guzzo, Carlie Phillips, and Briannon O'Connor \\ Department of Psychology, State University of New York at Buffalo
}

\begin{abstract}
Behavioral parent training is an efficacious treatment for attention-deficit/hyperactivity disorder (ADHD). However, single-mother households are at high risk for poor outcomes during and following behavioral parent training. This study randomly assigned cohorts of 120 single mothers of children (ages 5-12 years) with ADHD to a waitlist control group, a traditional behavioral parent training program, or an enhanced behavioral parent training program - the Strategies to Enhance Positive Parenting (STEPP) program. Intent-to-treat analysis demonstrated benefits of participating in behavioral parent training, in general, and the STEPP program more specifically at immediate posttreatment on child and parental functioning. Moreover, the STEPP program resulted in increased engagement to treatment. However, results indicated that behavioral parent training does not normalize behavior for most children and treatment gains are not maintained.
\end{abstract}

Behavioral parent training (BPT) has long been identified as an efficacious treatment approach for children with attention-deficit/hyperactivity disorder (ADHD), oppositional defiant disorder (ODD), and conduct disorder (CD; Brestan \& Eyberg, 1998; Pelham, Wheeler, \& Chronis, 1998). However, the extant litera-

Anil Chacko is now at the Department of Psychology, Queens College, City University of New York and the Department of Psychiatry, Mount Sinai School of Medicine. Brian T. Wymbs is now at the Department of Psychiatry, Western Psychiatric Institute and Clinic. Michelle S. Swanger-Gagne is now at the Educational Psychology Department, University of Nebraska-Lincoln. Erin Girio is now at the Department of Psychology, Ohio University. Support for this study was provided to the first author through a National Institutes of Mental Health, Pre-doctoral National Research Service Award (NRSA; 1 F31 MH071090-01A1), a New York State/Graduate Student Professional Development Award, a Society for a Science of Clinical Psychology Dissertation Award, a Melissa Institute for Violence Prevention and Treatment Dissertation Award, a Society for Clinical Child and Adolescent Psychology Graduate Student Research Award, and a University at Buffalo, College of Arts and Sciences Dissertation Award.

Correspondence should be addressed to Anil Chacko, Department of Psychology, Queens College, CUNY, 65-30 Kissena Boulevard, Flushing, NY 11367. E-mail: anil.chacko@qc.cuny.edu ture indicates that high levels of adversity (e.g., parental psychopathology, high levels of stress) often place families at risk for poor attendance and engagement during BPT and limited benefits from BPT (Chronis, Chacko, Fabiano, Wymbs, \& Pelham, 2004; Miller \& Prinz, 1990). Single mothers experience multiple adversity factors that impact their involvement in BPT, including higher rates of depression and stress as well as less social support (Cairney, Boyle, Offord, \& Racine, 2003). These parents are also more likely to have greater practical barriers to treatment participation (Kazdin, Holland, Crowley, \& Brenton, 1997; Kazdin \& Wassell, 2000) and maladaptive cognitions regarding treatment and their child (Nock \& Kazdin, 2001), resulting in additional difficulties faced by these families during BPT. Thus, it is not surprising that single-mother families are less likely to enroll in BPT (Cunningham et al., 2000), complete BPT (Kazdin \& Mazurick, 1994; Kazdin, Mazurick, \& Bass, 1993), improve following BPT (Dumas \& Wahler, 1983; Webster-Stratton \& Hammond, 1990), and maintain treatment gains over time (Bagner \& Eyberg, 2003; Webster-Stratton, 1985). Clearly, as suggested by leading BPT researchers, "one 
of the most important directions for the successful growth of BPT in the next decade is the development of effective treatments for single-parent, socially isolated families" (Dadds \& McHugh, 1992, p. 252).

Given the obvious need, the dearth of empirical studies on the enhancement of BPT for single mothers is surprising. Only three relatively small BPT studies have been conducted with this population - one with single mothers of children with elevated levels of behavior problems (i.e., Pfiffner, Jouriles, Brown, Etscheidt, \& Kelly, 1990), one with single parents (mothers and fathers) of children with ODD/CD (Dadds \& McHugh, 1992), and finally one study investigating the feasibility and preliminary efficacy of the Strategies to Enhance Positive Parenting (STEPP) program targeting single mothers of children diagnosed with ADHD (Chacko et al., 2008).

Pfiffner and colleagues (1990) randomly assigned 13 single mothers of children with elevated levels of behavioral problems to either traditional BPT or traditional BPT plus a problem-solving treatment. Although both treatment groups experienced significant reductions in parent-reported and observed child disruptive behavior, the BPT plus problem-solving treatment group experienced higher rates of clinically significant improvement at 4-month follow-up. This study provided preliminary support for BPT, as well as problem-solving as an adjunctive treatment to BPT, for single mothers of children with behavioral difficulties.

Dadds and McHugh (1992) evaluated the efficacy of an adjunctive social support intervention with BPT for 22 single parents (both mothers and fathers) of children diagnosed with ODD or CD. The social support intervention was aimed at improving the ability of important individuals in the lives of single parents (allies) to provide social support. These allies partnered with single parents, problem-solved, and identified solutions for parenting difficulties. Results of this randomized clinical trial demonstrated that the BPT-alone and the BPT plus ally support intervention resulted in improvements for both groups at posttreatment and follow-up, but no incremental benefits for the BPT plus ally support group were found.

To our knowledge, only one small, uncontrolled study has investigated the efficacy of an enhanced BPT intervention targeting single mothers of children specifically diagnosed with ADHD, ODD, or CD (Chacko et al., 2008). Chacko and colleagues evaluated the efficacy of the STEPP program, which included several adjunctive components addressing key areas influencing treatment engagement and outcomes for single mothers participating in BPT. In particular, the STEPP program focused on enhancements to the format, delivery, and content of traditional BPT including (a) an enhanced intake procedure that addressed practical barriers to treatment participation, maternal cognitions regarding expectations for treatment, and attributions regarding their children's behavior; (b) incorporating a subgroup, coping-modeling, problem-solving format within the traditional large group format to improve social support between parents and to increase participation among parents; and (c) incorporation of a systematic, problem-solving treatment to address parent-initiated problems. These additions to traditional BPT were included to address several major areas identified in the literature as being important to target with multiply stressed, single mothers: practical barriers to participation, maladaptive cognitions regarding their child and treatment, depression, social support, and life stressors (Chronis et al., 2004; Miller \& Prinz, 1990).

Results of this pilot study indicated that the STEPP program reduced problematic child behavior; improved parental stress and psychopathology; and resulted in high rates of treatment attendance, completion, and consumer satisfaction with the program. However, results also indicated that the STEPP program had less impact on children's overall levels of functional impairment and resulted in relatively small effect size findings across most measures. Results of the pilot study were encouraging but indicated a need to improve the potency and delivery of certain aspects of the STEPP program.

Based on quantitative and qualitative data gathered from the pilot study, the STEPP program was adapted. In particular, changes were made to the intensity, content, and activities of the STEPP program that may potentially lead to further improvements in outcomes. First, it was hypothesized that the intensity of the STEPP program was too low, particularly given the difficulties commonly experienced by the target population. Thus, the STEPP program was extended from nine $1 \frac{1}{2}$-hr sessions to nine $2 \frac{1}{2}$-hr sessions, thereby increasing the total therapeutic contact within the STEPP program by $9 \mathrm{hr}$. In addition, single mothers in the focus group indicated needing further support in implementing parenting skills covered during the treatment sessions. To enhance parenting skill acquisition, modifications were made to the concurrent children's social skills program. A modification was made such that during certain sessions, single mothers observe paraprofessionals modeling the use of the parenting skill with their child in the children's social skills program. Subsequently, single mothers would implement the parenting skill with their child in the social skills group with feedback provided by the therapist and other members of the parent's subgroup when the interaction was completed. Finally, given the feedback regarding the difficulty in implementing an incentive system at home, a within treatment incentive system was developed that focused on children earning incentives based on meeting both within-session and weekly, home-based behavioral goals. This would 
provide an opportunity for single mothers to observe how these incentive systems worked with their child and to have a consistent means of delivering rewards during the course of the STEPP program, and this would allow children in the STEPP program an opportunity to be consistently rewarded for attaining treatment goals at home and within-session.

This article reports results from a clinical trial designed to investigate the efficacy of the updated STEPP program. Specifically, the STEPP program was compared to a traditional BPT program and a waitlist control group with single-mother families of children diagnosed with ADHD. This study had three specific aims: (a) to compare single mothers assigned to active treatment (i.e., STEPP or the traditional BPT program) to a waitlist control group to determine the acute efficacy and 3-month follow-up benefit of BPT; (b) to determine whether the STEPP program enhanced acute and 3-month follow-up benefits relative to traditional BPT; and (c) to examine whether the STEPP program resulted in greater attendance, engagement, and consumer satisfaction compared to traditional BPT.

It was hypothesized that children with ADHD and their single mothers receiving an active BPT program would benefit from treatment relative to the waitlist control group. In addition, it was hypothesized that the STEPP program would result in greater reductions in child ODD symptoms and impairment, parental stress, maternal depressive symptoms, and observed negative parenting behavior, as well as increased frequency of observed positive parenting behavior compared to the traditional BPT program at posttreatment. It was hypothesized that the STEPP program would result in increased single-mother attendance, engagement, and consumer satisfaction. Last, it was hypothesized that gains observed following the STEPP program would maintain at the 3-month follow-up assessment compared to the traditional BPT program.

\section{METHOD}

\section{Participants}

Between September 2002 and March 2005, singlemother families were recruited for this study through radio advertisements, mailings, and school referrals. Mothers were required to be the primary caregiver and residing without a significant other (e.g., child's father, boyfriend, fiancé); however, mothers were included in this study if they resided with other individuals (e.g., parents, siblings, roommates). Mothers were not excluded from participation for the presence of any psychiatric conditions. Children were required to be between 5 to 12 years old at the start of treatment and were required to meet diagnostic criteria for ADHD (any type). The family met with study staff to conduct a formal assessment for a diagnosis of ADHD prior to enrolling in the study. As recommended for evidencebased assessment of ADHD (Pelham, Fabiano, \& Massetti, 2005), ADHD diagnosis was determined through completion of parent and teacher rating scales of Diagnostic and Statistical Manual of Mental Disorders (DSM) symptoms (i.e., Disruptive Behavior Disorder rating scale; Pelham, Gnagy, Greenslade, \& Milich, 1992), completion of semistructured interviews with the parent (Disruptive Behavior Disorder semistructured parent interview; Pelham, 2002), and assessment of cross-situational impairment through completion of parent and teacher rating scales (Impairment Rating Scale; Fabiano et al., 2006). Children were included in this study if they were receiving medication for their ADHD symptoms. For children who were receiving medication, parents were asked to maintain the type and dose of medication for the duration of the study and report any changes in medication status to the research study team (see Table 1). Similarly, for children who were not receiving medication, parents were asked

TABLE 1

Participant Characteristics for STEPP, Traditional BPT, and Waitlist Control Groups

\begin{tabular}{|c|c|c|c|}
\hline & STEPP & Traditional $B P T$ & Waitlist \\
\hline Child Age in Years $(M, S D)$ & $7.36(1.86)$ & $8.17(2.42)$ & $8.02(2.15)$ \\
\hline Child Sex & $77 \%$ male & $66 \%$ male & $69 \%$ male \\
\hline Single Mother Age (Years) & $34.05(8.27)$ & $36.77(8.56)$ & $35.25(8.65)$ \\
\hline Single Mother Education (Years) & $13.84(1.66)$ & $14.28(1.95)$ & $14.22(1.88)$ \\
\hline Child Race/Ethnicity & $\begin{array}{l}52 \% \text { Caucasian, } 27 \% \\
\text { African American, } 8 \% \\
\text { Latino, } 13 \% \text { biracial }\end{array}$ & $\begin{array}{l}56 \% \text { Caucasian, } 18 \% \\
\text { African American, } 13 \% \\
\text { Latino, } 13 \% \text { biracial }\end{array}$ & $\begin{array}{l}52 \% \text { Caucasian, } 18 \% \\
\text { African American, } 17 \% \\
\text { Latino, } 13 \% \text { biracial }\end{array}$ \\
\hline $\begin{array}{l}\text { Child ODD/CD } \\
\text { Comorbidity Status }\end{array}$ & $67.5 \%$ ODD, $12.5 \% \mathrm{CD}$ & $70 \%$ ODD, $10 \% \mathrm{CD}$ & $72.5 \%$ ODD, $7.5 \% \mathrm{CD}$ \\
\hline Percent Medicated & $40 \%$ & $35 \%$ & $37.5 \%$ \\
\hline
\end{tabular}

Notes. There were no significant differences between groups on any variables in the table. Some percentages sum to greater than $100 \%$ due to rounding error. STEPP = Strategies to Enhance Positive Parenting; BPT = Behavioral Parent Training; ODD = oppositional defiant disorder; $\mathrm{CD}=$ conduct disorder. 
to maintain this medication status for the duration of the study and report any changes in medication status to the research study team. In addition, information on medication status was collected at each of the three assessment points to determine changes in medication regimens. Families were excluded if the child had an IQ of less than 80 , if the child was diagnosed with a pervasive developmental disorder, or if there was evidence of psychosis. The final sample consisted of 120 children with ADHD and their single mothers.

\section{Procedures}

Single mothers' consent and child assent was obtained before the initial intake. The study procedures were approved by the University at Buffalo's Social and Behavioral Sciences Institutional Review Board. To maximize treatment group size, active treatment (i.e., traditional BPT and STEPP program) was randomly assigned to semester (i.e., fall or spring semester). Single mothers were randomly assigned within semester to either the active treatment group for that semester (traditional BPT or STEPP) or the waitlist control group. Treatment conditions included (a) a 9-week, traditional BPT group; (b) the 9-week STEPP program; or (c) a 9 -week waitlist control group. Forty participants were assigned to each study condition. Characteristics of the BPT group participants and the waitlist control group participants are detailed in Table 1 . The groups did not differ on any of the demographic variables.

Traditional BPT. The traditional BPT program is a manualized, 9-week BPT program held for $2 \frac{1}{2} \mathrm{hr}$ each week that was developed for this study based on the work of empirically supported BPT interventions (Barkley, 1997, Cunningham, Bremner, \& Secord, 1998; Wells et al., 1994). Single mothers engaged in a collaborative, large-group format to discuss and learn about effective parenting strategies (e.g., positive attending, planned ignoring, incentive systems). Given the range in children's age, therapists tailored treatment content to be appropriate to each parent's child's developmental level. For instance, discussions of positive attending for parents of younger children would be discussed within the context of play, whereas for parents of older children positive attending would be discussed within the context of watching TV, reading magazines, or having discussion between the parent and the child. Furthermore, sessions included videotapes of parenting errors whereby single mothers identified these errors and then formulated alternative parenting strategies. Furthermore, therapists facilitated group discussions by asking questions to encourage single mothers to make adaptive attributions about the effects of their parenting on their children's behavior. Therapists modeled the parenting techniques with role-plays by single mothers. Single mothers were assigned weekly homework assignments based on the content of the session.

During the program, children participated in a concurrent traditional, group-based social skills program (Cunningham et al., 1998). Children were divided into two groups based on the developmental level of the child. Typically, children between the ages of 5 to 8 formed one group, and children between the ages of 9 to 12 formed another group. Children were supported in the acquisition of key social skills used in peer contexts (e.g., cooperation, validation) through didactic training, modeling, role-playing and ongoing support of the skills through age-appropriate small-group games.

STEPP program. Like traditional BPT, the STEPP program was a manualized, 9-week program held for $2 \frac{1}{2}$ $\mathrm{hr}$ each week, which included a collaborative largegroup format, identical evidence-based BPT content, identical order of presentation of BPT content, identical videotaped vignettes, therapist-facilitated questions, group discussions, modeling, and role-plays by parents. Children participated in a group social skills program.

The STEPP program, however, also includes several enhancements to the format, delivery, and content of traditional BPT based on the extant literature (Chacko et al., 2008). First, the STEPP program incorporates an enhanced intake procedure that improves parents' motivation to engage in treatment, addressing possible practical barriers to treatment participation, and addressing maternal cognitions regarding expectations for treatment and attributions regarding their child's behavior (McKay, Stoewe, McCadam, \& Gonzales, 1998; Morrissey-Kane \& Prinz, 1999; Nock \& Kazdin, 2005; Prinz \& Miller, 1996). For instance, open-ended questions were asked of single mothers regarding their expectations about their as well as their child's involvement in treatment (e.g., What role do you think you will have in treatment? In what way do you think your child will be involved in treatment?). Single mothers were also asked open-ended questions regarding their expectations about the rate and potency of treatment-related improvements for their child (e.g., How fast do you expect to observe improvements in your child's behavior?) and about their attributions regarding locus of control of their child's behavior (e.g., What do you think causes your child to misbehave?) and the affect of their parenting (e.g., In what ways have you seen you parenting make a difference?). Misconceptions/inappropriate cognitions regarding these issues were discussed and clarified with the single mother during the intake. Last, practical barriers (e.g., child care, transportation) to ongoing involvement were addressed and solutions to these barriers were developed during the intake. 
Another modification of the STEPP program was to incorporate a subgroup, coping-modeling, problemsolving format within the traditional large-group format to improve social support between parents and to increase participation among parents (Cunningham, Davis, Bremner, Dunn, \& Rzasa, 1993). Also, the STEPP program incorporates a systematic, problemsolving treatment method (D'Zurilla \& Nezu, 1999) to address parent-initiated problems (e.g., time management, conflicts with relatives) that may either interfere with their parenting or affect parents' psychosocial functioning (Kazdin \& Whitley, 2003; Pfiffner et al., 1990; Prinz \& Miller, 1994; Spaccarelli, Cotler, \& Penman, 1992). In addition, the STEPP program incorporates parent-child interactions within the children's social skills group to enhance parenting skill acquisition and a child motivation enhancement within the children's social skills group to provide children incentives for attaining withinsession and home-based behavioral goals.

\section{Measures}

ADHD/ODD symptoms. The DSM-IV-TR (4th ed., text rev.; American Psychiatric Association, 2000) ADHD and ODD symptoms were measured using the Disruptive Behavior Disorders (DBD) rating scale. The DBD scale is a 45-item measure that asks parents to rate DSM symptoms of ADHD, ODD, and CD on a 4-point Likert scale (i.e., not at all, just a little, pretty much, or very much), with higher scores indicating more problems. For this study, the average scores for the DSM Inattentive symptoms, DSM HyperactiveImpulsive symptoms, and DSM ODD symptoms were used. Support for the scales reliability and validity have been reported in past samples, with acceptable levels of internal consistency $(\alpha=.82)$ and concurrent correlations with other measures used for diagnosis of ADHD and ODD (e.g., DISC; $r=.62$ ) being reported (Pelham et al., 2005). The DBD rating scale has also been reported to be sensitive to treatment effects in multiple studies (see Pelham et al., 2005, for a review).

Functional impairment. Parent ratings of problem severity and need for treatment in key functional domains were measured using the Impairment Rating Scale (IRS). The IRS measures impairment across domains of functioning as well as overall need for treatment. Parents place an " $\mathrm{X}$ " on a 7-point visual analogue scale to signify their child's functioning along a continuum of impairment that ranges from 0 (Not a problem at all. Definitely does not need treatment or special services) to 6 (Extreme problem. Definitely needs treatment and special services). IRS items that measures the impact of the child's problems on peer relationships, relationship with their parent, family functioning, and overall impairment were used in the analyses. Testretest reliability has been reported $(r=.82-.95$ over 2 months) and concurrent validity with the Parent CGAS $(r=.55-.73)$. The IRS is sensitive behavioral and pharmacological effects of treatment, correlate with behavioral observations and frequency counts of behavior, and predictive of mental health treatment use in children (Fabiano et al., 2006).

Parenting. Observed parenting behavior was measured using the Dyadic Parent-Child Interaction Coding System (DPICS; Parenting Clinic, 2000; Robinson \& Eyberg, 1981). The validity of the DPICS has been evaluated through multiple studies (e.g., Robinson \& Eyberg, 1981). For the purpose of this study, the composite scores for Positive Parenting (includes praise, positive affect, and physical positive) and Negative Parenting (includes negative command, critical statements, and physical negative) were used.

The parent-child interactions coded using the DPICS were based on standard interactions developed for use with the DPICS (Parenting Clinic, 2000; Robinson \& Eyberg, 1981). The parent-child interaction consisted of three 5-min segments, totaling a 15 -min observation period. In the first 5-min segment, the parent was instructed to play/interact along with their child and allow the child to take the lead. During the second 5-min segment, the parent was instructed to play/ interact with their child but the parent was required to take the lead. During the last 5-min segment, the parent was instructed to have their child clean up the toys.

A team of two independent coders were trained in the use of the DPICS until $80 \%$ agreement was attained. Overall reliability was computed by calculating Agreements/Agreements + Disagreements (Parenting Clinic, 2000; Robinson \& Eyberg, 1981). Reliability checks were conducted to maintain an acceptable level of agreement among coders. The primary coder observed and coded all of the tapes and $40 \%$ of these tapes were coded by a second coder to assess interrater reliability. Interobserver agreement coefficients were .93 for Positive Parenting and .91 for Negative Parenting.

Parental psychosocial adjustment. The Beck Depression Inventory (BDI) is a 21-item self-report measure used to assess maternal depressive symptoms over the preceding 2-week period. The BDI is scored from 1 to 4, with higher total scores on the BDI indicating a greater degree of depression. The BDI is highly correlated with clinical ratings of depression $(r=.72)$ and has been shown to have high internal consistency in both clinical and nonclinical samples, with mean 
coefficient alphas of .86 and .81 , respectively (Beck, Steer, \& Garbin, 1988).

The Parenting Stress Index-Short Form (PSI-SF; Abidin, 1995) is a 36-item self-report measure used to assess parenting stress in three domains-Parental Distress, Parent-Child Dysfunctional Interaction, and Difficult Child. The PSI-SF is measured along a 5-point scale from 1 (strongly disagree) to 5 (strongly agree), with higher total scores indicate greater levels of parenting stress. High levels of internal consistency $(\alpha=.83)$ and 1-year stability $(r=.75)$ have been reported (Haskett, Ahern, Ward, \& Allaire, 2006). The PSI-SF correlates with parental perceptions of child adjustment and observed parent and child behavior adjustment (Haskett et al., 2006).

Therapy process and engagement. The percentage of sessions attended by the single mother and by the child, the percentage of sessions attended on time, and the percentage of homework assignments completed were used as a measure of parent and child engagement.

Consumer satisfaction. The Therapy Attitude Inventory (TAI; Brestan, Jacobs, Rayfield, \& Eyberg, 1999) is an index of consumer satisfaction for participants in parent training. Items are rated on a scale from 1 (indicating treatment dissatisfaction or lack of improvement) to 5 (indicating satisfaction with treatment and improvement). The TAI is comprised of two factors: satisfaction with treatment outcome and satisfaction with treatment process. The TAI has been shown to have excellent consistency $(\alpha=.91)$ and stability over a 4-month period (.85). In addition, external validity was demonstrated by moderate correlations (.36-.49) between TAI scores and changes in observed and parent self-report of children's behavior after treatment (Brestan et al., 1999).

Treatment integrity and fidelity. Each intake session was audiotaped and each treatment session was videotaped. An independent coder coded the tapes for intake and treatment session content using standardized checklists. Raters (study participants and independent raters) assessed treatment fidelity and therapist skill/behavior. High levels of treatment fidelity and therapist competence were obtained for the traditional BPT and the STEPP programs (data on treatment fidelity and therapist competence data are available from the first author by request). All measures, except for consumer satisfaction (TAI), were collected at pretreatment and posttreatment (immediately following the completion of treatment). Furthermore, at 3-month follow-up, the DBD Rating Scale and the IRS were also completed.

\section{RESULTS}

Random-effects regression (RR) using SAS PROC MIXED procedure (SAS v.8.2) was used for primary intent-to-treat analyses for the specific aims of this study. Complete data were collected on $98 \%(n=118)$ of participants at posttreatment and 95\% $(n=115)$ participants at follow-up. Two sets of contrasts were tested for the three specific aims of this study. First, analyses compared the waitlist group to the combined BPT group (i.e., STEPP and traditional BPT groups). Second, analyses compared the STEPP group with the traditional BPT group. For each dependent variable, the first-order effect of time of assessment (i.e., pretreatment to posttreatment) was assessed, as well as firstorder effect of each contrast (i.e., Combined BPT vs. waitlist or STEPP vs. traditional BPT). When applicable, two additional first-order effects of time of assessment (posttreatment to follow-up and pretreatment to follow-up) were assessed to determine if maintenance/ improvements in functioning occurred past the acute effects of treatment. Group $\times$ Time interactions were examined to determine if improvement in functioning over time was differentially associated with treatment group. A conservative alpha level (i.e., $p<.01$ ) was adopted for these analyses.

The clinical significance of treatment effects was analyzed to determine whether a child moved out of the clinical range on the DBD Rating Scale (ADHD and ODD score) and the Impairment Rating Scale-Overall Impairment domain using available norms. The clinical cutoff for the DBD ADHD and ODD score is any score less than or equal to 1 (Swanson et al., 2001). The clinical cutoff for the Impairment Rating Scale-Overall Impairment domain is any score less than three (Fabiano et al., 2006). A conservative approach to data analysis was used whereby any child with a pretreatment score below the clinical cutoff (and therefore not meeting clinical status at pretreatment) was classified as "unimproved" for the purpose of these analyses. Last, effect size estimates (Cohen's $d$ ) were computed for each outcome measure.

\section{Efficacy of BPT (Combined Traditional BPT and STEPP) for Single Mothers}

$R R$ analyses. Acute benefits of the combined BPT group compared to the waitlist control group were observed on several outcomes (see Table 2). RR analyses indicate significant benefits of combined BPT on ODD symptoms, $t(204)=2.65, p<.009$, as well as parent, $t(200)=3.50, p<.0006$; family, $t(200)=3.78$, $p<.0002$; and overall, $t(199)=4.30, p<.0001$, impairment. Positive, $t(117)=2.65, p<.009$, and negative, 


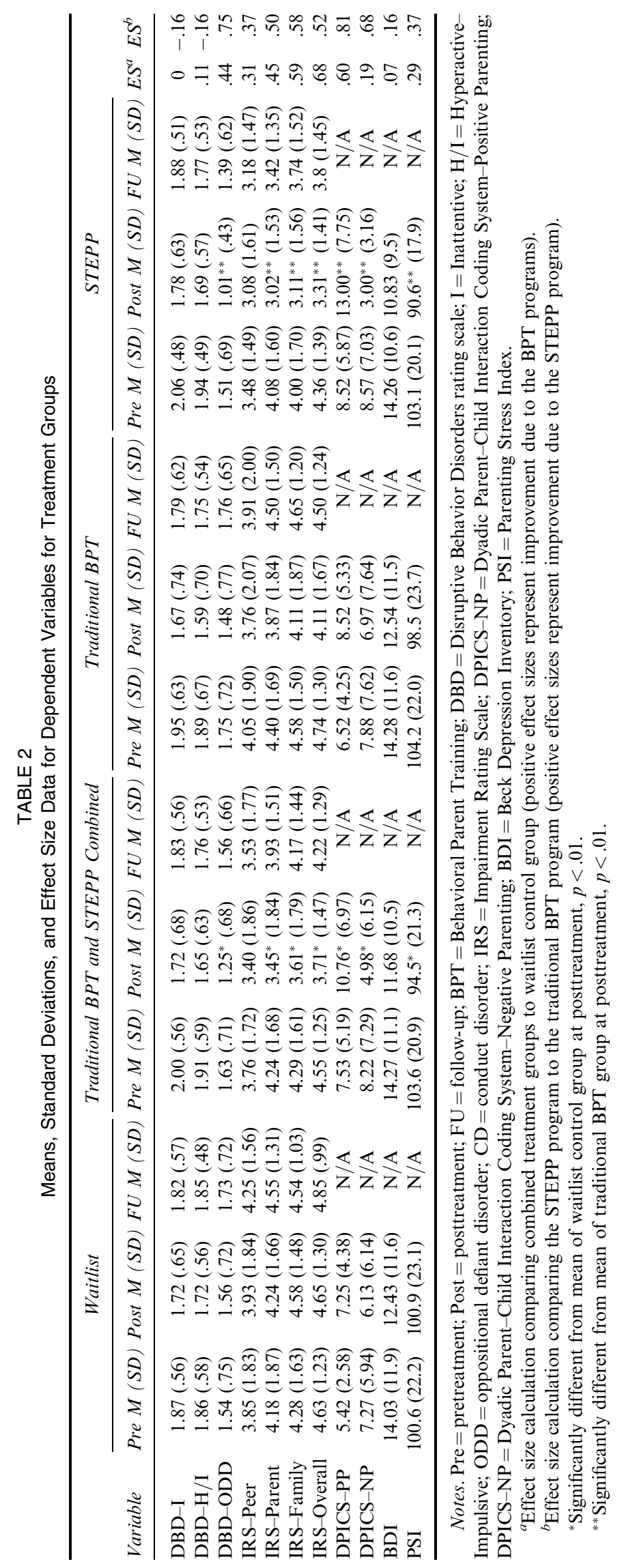


$t(117)=2.65, p<.009$, observed parenting also significantly improved with the combined BPT groups. Last, parenting stress, $t(117)=4.15, p<.001$, was significantly reduced with the combined BPT groups. No maintenance of treatment gains were observed following completion of the combined BPT groups.

Clinical significance. Fifteen percent and 20\% of children assigned to the combined BPT group were rated as below the clinical cutoff on ADHD and ODD symptoms, respectively, versus $7.5 \%$ and $10 \%$ of children assigned to the waitlist control group. On ratings of IRS overall severity, $15 \%$ were below the clinical cutoff in the combined BPT group versus $5 \%$ in the waitlist control group. These differences in rates of normalization were analyzed using logistic regression. Group status (combined BPT or waitlist control group) was entered as a predictor and normalization (yes or no) was entered as a dependent variable. Although more children and parents were normalized in each domain in the combined BPT group, no analyses were statistically significant.

Effect size. Effect size calculations were conducted for each outcomes measure between the combined BPT and waitlist control groups. Effect sizes ranged from no effect to a moderate effect (0-.68), with the mean effect size equaling 0.36 , with a majority of effect sizes indicating improvement because of the combined BPT treatment (see Table 2).

\section{STEPP Versus Traditional BPT}

$R R$ analyses. Acute benefits of the STEPP program compared to traditional BPT were observed on several outcomes (see Table 2). RR analyses indicate significant benefits of the STEPP program on child ODD symptoms, $t(204)=-2.65, p=.009$, as well as parent, $t(200)=$ $-3.55, p=.0005$; family, $t(200)=3.23, p<.001$; and overall, $t(199)=3.20, p<.002$, impairment. Positive, $t(117)=-4.72, p<.001$, and negative, $t(117)=2.65$, $p<.009$, observed parenting also significantly improved with the STEPP program. Last, parenting stress, $t(117)=4.15, p<.001$, was significantly improved with the STEPP program. No maintenance of treatment gains were observed following STEPP or BPT.

Clinical significance. Twenty percent of children assigned to the STEPP group were rated as below the clinical cutoff on ADHD symptoms versus $10 \%$ of children assigned to the traditional BPT group. Twenty-five percent of children assigned to the STEPP group were rated as below the clinical cutoff on ODD symptoms versus $15 \%$ of the children assigned to the traditional BPT group. Twenty percent were below the clinical cutoff for the IRS overall severity scale following the STEPP group versus $10 \%$ in the traditional BPT group. These differences in rates of normalization were analyzed using logistic regression. Again, no analyses were statistically significant.

Effect sizes. Effect sizes ranged from a negative effect to a large effect $(-.16-.81)$, with the mean effect size equaling 0.44 , with a majority of effect sizes indicating improvement due to the STEPP program (see Table 2).

Therapy process measures. Therapy process measures (e.g., percentage of sessions attended by the single mother, percentage of sessions attended by the child, percentage of sessions attended on-time, and percentage of homework completed) were analyzed with a multivariate analysis of variance, using treatment group as the between-subject factor (Group: STEPP, traditional

TABLE 3

Means and Standard Deviations for STEPP Versus Traditional BPT Therapy Process and Consumer Satisfaction Measures

\begin{tabular}{|c|c|c|c|c|c|}
\hline & \multicolumn{2}{|c|}{$S T E P P$} & \multicolumn{2}{|c|}{ Traditional BPT } & \multirow[b]{2}{*}{$E S$} \\
\hline & $M$ & $S D$ & $M$ & $S D$ & \\
\hline Single Mother $\%$ of Sessions Attended & $77.2^{*}$ & 24.9 & 39.4 & 35.6 & 1.06 \\
\hline Child $\%$ of Sessions Attended & $74.4^{*}$ & 27.9 & 39.4 & 34.9 & 1.00 \\
\hline Single Mother $\%$ of Sessions Attended On-Time & 52.6 & 30.4 & 44.5 & 37.8 & .21 \\
\hline Single Mother \% Homework Completed & $48.0^{*}$ & 28.6 & 28.5 & 26.2 & .74 \\
\hline TAI-SWO & $24.18^{*}$ & 3.02 & 20.20 & 2.35 & 1.69 \\
\hline TAI-SWP & $16.36^{*}$ & 2.03 & 14.12 & 2.09 & 1.07 \\
\hline
\end{tabular}

Notes. STEPP $=$ Strategies to Enhance Positive Parenting; BPT $=$ Behavioral Parent Training; ES $=$ effect size (Positive effect sizes represent improvement because of the STEPP program); TAI-SWO = Treatment Attitude Inventor-Satisfaction with Outcome; TAI-SWP = Treatment Attitude Inventory-Satisfaction with Process.

${ }^{*}$ Significantly different from mean of traditional BPT, $p<.01$. 
BPT). The omnibus test yielded a significant effect for group, $F(3,75)=6.37, p=.0007$. Univariate effects were significant for single-mother attendance $(p<.0001)$, child attendance $(p<.0001)$, and homework compliance $(p<.0001)$. The results indicate that single mothers in the STEPP program were more likely to attend treatment and were more likely to complete homework assignments. Moreover, children in the STEPP program were also more likely to attend treatment. Effect sizes were calculated for therapy process measures with effect sizes ranging from 0.21 to 1.06 , indicating beneficial effects of the STEPP program (see Table 3).

Consumer satisfaction. Scores on the TAI for the STEPP and traditional BPT program were analyzed using an independent samples $t$ test for the "satisfaction with outcome" and "satisfaction with treatment progress" factors. Results indicated beneficial effects of the STEPP program on the satisfaction with outcome factor, $t(64)=4.18, p<.001$, and a significant effect on the satisfaction with process factor, $t(64)=3.05$, $p=.003$. Effect sizes equaled 1.07 for the satisfaction with process variable and 1.69 for the satisfaction with outcome variable, indicating beneficial effects of the STEPP program (see Table 3 ).

\section{DISCUSSION}

To our knowledge, this was the first controlled clinical trial to specifically assess the efficacy of BPT for single mothers of children with ADHD. The intent-to-treat analyses demonstrated the acute efficacy of BPT as an intervention for single mothers of children with ADHD compared to a waitlist control group. Participation in BPT resulted in acute improvements on measures of oppositional defiant behavior, parent-child and familyrelated impairment, observational measures of parenting behavior, and parenting stress; findings that parallel other studies of BPT (Brestan \& Eyberg, 1998; Nock, 2003; Pelham et al., 1998). Effect-size calculations also demonstrated the benefit of BPT compared to the waitlist control group. Of importance, however, BPT did not yield significant improvements relative to the waitlist control group on several measures including symptoms of ADHD, parent-rated peer-related impairment, and maternal depression. BPT for single mothers did not normalize functioning for the majority of children with ADHD and benefits were generally not maintained at 3-month follow-up.

A similar pattern of findings were observed when comparing the STEPP program to the traditional BPT program. Specifically, relative to traditional BPT, the STEPP program resulted in acute improvements on measures of oppositional defiant behavior, parent-child and family-related impairment, observational measures of parenting behavior, and parenting stress. Moreover, the STEPP program had significant positive effects on single mother and child attendance, homework compliance, and consumer satisfaction. No differences were observed between the two treatment conditions on symptoms of ADHD, parent-rated peer-related impairment, and maternal depression. Moreover, the STEPP program resulted in clinically significant benefits for only a minority of children, and maintenance of gains were not observed following completion of treatment.

The statistical significance and effect size data regarding the STEPP program relative to traditional BPT on immediate outcomes are notable given the limited benefits of traditional BPT for single mothers reported in the extant literature (Lundahl, Risser, \& Lovejoy, 2006). Enhancements delivered via the STEPP program likely increased opportunities to affect key areas related to both child behavior and parental functioning. For example, the STEPP program sought to increase opportunities for parents to improve their parenting behavior through the within-session parent-child interactions. In addition, the adjunctive problem-solving component may have also likely influenced parents' ability to manage life stressors that may have lead to improvements in parental stress, which in turn may have resulted in further improvements in parenting behavior and, ultimately, child disruptive behavior. Moreover, the child motivation enhancement delivered in the STEPP program may have also likely impacted child behavior by reinforcing children's efforts in changing their own behavior during sessions and at home. Collectively, we contend that greater improvements in child behavior, functional impairment, and parental stress were observed in families assigned to the STEPP program because of these enhancements.

Arguably, the most marked and significant finding in this study relates to the effects of the STEPP program on attendance, engagement, and satisfaction with treatment. Data indicated that single mothers in the STEPP program attended significantly more treatment sessions compared to single mothers in the traditional BPT program. Of importance, the attendance rate in the STEPP program group compares favorably to other studies that have focused on enhancing attendance to BPT (e.g., Nock \& Kazdin, 2005; Prinz \& Miller, 1994). However, unlike these studies, which provided enhancements to improve retention in BPT for a general sample of families, our study targeted a population at high risk for poor attendance to BPT.

Although benefits were observed on key outcomes, BPT interventions did not result in improvements in several areas. First, no significant effects were found on parent-reported ADHD symptoms. Perhaps the lack 
of treatment effects on parent reports of ADHD symptoms is due to the nature of BPT in this study, which explicitly addressed improving impairment (e.g., disruptive behavior) rather than ADHD symptoms. Alternatively, studies demonstrating effects of BPT on ADHD typically have incorporated content related to ADHD symptoms (e.g., Anastopoulos, Shelton, DuPaul, \& Guevremont, 1993). However, the STEPP program's focus on targeting impairment is because impairment in daily life functioning is related to long-term functioning for children rather than psychiatric symptoms of ADHD (see Pelham \& Fabiano, 2008; Pelham et al., 2005, for a discussion).

In addition, no significant treatment effects were observed on peer-related impairment. One explanation for this finding may be that parents are not the best reporters of their child's peer relationships (Renk \& Phares, 2004). In addition, it may be that the type of intervention implemented in this study was not frequent enough or conducted over a sufficient period of time to improve peer relationship difficulties in children with ADHD. Positive peer relationships are critical for long-term adjustment in children, but existing treatment modalities, including intensive behavioral and pharmacological interventions, have not been shown to ameliorate the long-term difficulties children with ADHD experience in peer relationships (Hoza et al., 2005). Clearly, given the increasing importance of peer relationships for children over time, greater attention must be given to developing interventions that ameliorate the peer-relationship difficulties children with ADHD experience.

Despite the significant findings of the BPT program on parenting stress, maternal depressive symptoms did not improve relative to the waitlist control group. However, these findings are less surprising given that depression is related to many factors outside the parent-child context (Chronis, Pelham, Gnagy, Roberts, \& Aronoff, 2003). The STEPP program, which included a problemsolving component targeting a range of difficulties, did not provide specific benefits on ratings of depression. It may also be that the adjunctive problem-solving intervention was neither specific enough to nor administered intensively enough to improve depressive symptoms. In fact, studies that specifically address parental depression in BPT are often more specific, more intensive, and lengthier (e.g., Sanders \& McFarland, 2000).

The lack of significant findings on maintenance of treatment gains may be because of several issues. First, the small sample size of this study likely made it difficult to detect significant findings. Secondly, relying solely on reports of children's behavior from parents who are highly stressed is likely influenced by multiple factors aside from the actual behavior of their child (DeaterDeckard, 2004). Clearly, a larger sample size that includes multimethod, multi-informant methods would prove beneficial in more accurately determining whether maintenance of gains occur following BPT for singlemother families of children with ADHD. Last, and most likely, interventions that target high-risk groups should incorporate procedures to provide long-term, ongoing consultation and support (Bagner \& Eyberg, 2003; Eyberg, Edwards, Boggs, \& Foote, 1998). Clearly, given the multiple difficulties that single-mother families face in their own lives and in raising their child with ADHD, support following the completion of BPT should be immediate and frequent. If, how, and to what extent maintenance programs will be beneficial for highly stressed families is an important research endeavor.

Of note, a minority of single mothers in the BPT groups rated their child as below clinical cutoffs on ADHD or ODD symptoms or impairment, and these rates of reported normalization were not significantly different from rates reported by the waitlist control group. Although the findings on clinical significance of treatment were discouraging, the findings must be interpreted in light of the high-risk nature of single-mother families of children with clinical levels of disruptive behavior difficulties. For example, studies suggest that, compared to married mothers of children with ADHD, ODD, and/or CD, single mothers exhibit higher levels of stress in response to laboratory interactions with disruptive children, exhibit higher levels of overall parental stress, exhibit more ineffective parenting behavior, and have children who exhibit higher levels of disruptive behavior (Pelham, Lang, et al., 1998; Webster-Stratton, 1989). Collectively, single-mother families with children diagnosed with ADHD, ODD, and/or CD potentially face multiple challenges when participating in treatment. Multimodal treatment that addresses multiple issues, provided early in a child's life, and over a substantial period it is likely necessary to provide maximal benefit for these high-risk families.

\section{Implications for Research, Policy, and Practice}

Although the STEPP program offers clear advantages to traditional BPT, it is evident that the STEPP program requires further adaptation to maximize benefits of this intervention. First, although the STEPP program included multicomponent enhancements to traditional BPT, which resulted in greater attendance, homework compliance, and outcomes for single mothers, little can be stated regarding which of these components were necessary for these outcomes. Given that there are clear differences in time and costs for these individual components, it will be beneficial to determine which components are necessary and which do not provide incremental benefits beyond less intensive components. As suggested by others (Nock, 2003), 
there is a great need for research that elucidates the process of therapeutic improvements for children and parents in BPT.

Based on qualitative feedback from study participants and therapists, several recommendations for future iterations of the STEPP program are worth noting. First, although the subgroup-based format of the STEPP program was likely an important feature in enhancing social support between and learning among parents, feedback from parents suggested that for certain families, particularly those with greater number of risk factors, adjunctive individual sessions that focused on assisting families to tailor the content they learned during the group sessions would be necessary. Second, in corroboration with the quantitative data, parents noted a need for increasing the duration of both active treatment as well as a need for ongoing supportive booster sessions to maintain benefits. Finally, a majority of parents noted that additional assistance in working with school staff would be an important modification of the STEPP program. This suggestion is not surprising given that many children with ADHD experience significant difficulties in the school setting. Collectively, modifications to the duration, intensity, format, and focus of the STEPP program may result in significantly greater clinical improvements as well as long-term functioning for children with ADHD from high-risk backgrounds.

Clinically, it is clear that for high-risk samples, attention should be given to addressing issues of attendance and engagement to treatment. Thus, developing effective and engaging interventions should be a high priority for those intervening with high-risk samples. To date, including this study, the extant literature does support the use of addressing pretreatment barriers to engagement as well as incorporating problem-solving treatment during BPT as methods to enhance engagement, and outcomes (Kazdin \& Whitley, 2003; McKay, Stoewe, McCadam, \& Gonzales, 1996; McKay et al., 1998; Pfiffner et al., 1990; Prinz \& Miller, 1994). These components are relatively simple procedures and can be implemented as part of both group and individual BPT formats.

This study suggests, as well as numerous others (e.g., Swanson et al., 2001), that normalization of functioning for a majority of children with ADHD may be difficult to attain given the confluence of problems in these children and their families. This may be particularly the case when families are facing multiple adversities, such as single-mother families. Potentially, more comprehensive and intensive early preventive interventions for ADHD should be an important goal in order to alter the often problematic trajectories for these children and their families. It is likely; however, despite implementation of early and comprehensive intervention, parents of children with ADHD will likely require support during key developmental periods that may exacerbate difficulties for their children (e.g., transition from elementary to middle school). Developing and studying early, comprehensive, long-term intervention strategies must be a public health priority for children with ADHD from high-risk backgrounds.

\section{REFERENCES}

Abidin, R. R. (1995). Parenting stress index (3rd ed.). Odessa, FL: Psychological Assessment Resources.

American Psychiatric Association. (2000). Diagnostic and statistical manual of mental disorders (4th ed., text rev.). Washington, DC: American Psychiatric Association.

Anastopoulos, A. D., Shelton, T. L., DuPaul, G. J., \& Guevremont, D. C. (1993). Parent training for attention-deficit hyperactivity disorder: Its impact on parent functioning. Journal of Abnormal Child Psychology, 21, 581-596.

Bagner, D. M., \& Eyberg, S. E. (2003). Father involvement in parent training: when does it matter? Journal of Clinical Child and Adolescent Psychology, 32, 599-605.

Barkley, R. A. (1997). Defiant children: A clinician's manual for assessment and parent training (2nd ed.). New York: Guilford.

Beck, A. T., Steer, R. A., \& Garbin, M. G. (1988). Psychometric properties of the Beck Depression Inventory: Twenty-five years of evaluation. Clinical Psychology Review, 8, 567-571.

Brestan, E. V., \& Eyberg, S. M. (1998). Effective psychosocial treatments of conduct-disordered children and adolescents: 29 years, 82 studies, and 5, 272 kids. Journal of Clinical Child Psychology, 27, 180-189.

Brestan, E. V., Jacobs, J. R., Rayfield, A. D., \& Eyberg, S. M. (1999). A consumer satisfaction measure for parent-child treatments and its relation to measures of child behavior change. Behavior Therapy, 30, $17-30$.

Cairney, J., Boyle, M., Offord, D., \& Racine, Y. (2003). Stress, social support, and depression in single and married mothers. Social Psychiatry and Psychiatric Epidemiology, 38, 442-449.

Chacko, A., Wymbs, B. T., Flammer-Rivera, L., Pelham, W. E., Walker, K. S., Arnold, F., et al. (2008). A pilot study of the feasibility and efficacy of the Strategies to Enhance Positive Parenting (STEPP) program for single mothers of children with ADHD. Journal of Attention Disorders, 12, 270-280.

Chronis, A. M., Chacko, A., Fabiano, G. A., Wymbs, B. T., \& Pelham, W. E. (2004). Enhancements to the behavioral parent training paradigm for families of children with ADHD: Review and future directions. Clinical Child and Family Psychology Review, 7, $1-27$

Chronis, A. M., Pelham, W. E., Gnagy, E. M., Roberts, J. E., \& Aronoff, H. R. (2003). The impact of late-afternoon stimulant dosing for children with ADHD on parent and parent-child domains. Journal of Clinical Child and Adolescent Psychology, 32, 118-126.

Cunningham, C. E., Boyle, M., Offord, D., Racine, Y., Hundert, J., Secord, M., et al. (2000). Tri-ministry study: Correlates of schoolbased parent course utilization. Journal of Consulting and Clinical Psychology, 68, 928-933.

Cunningham, C. E., Bremner, R., \& Secord, M. (1998). COPE: The Community Parent Education Program: A School-Based Family Systems Oriented Workshop for Parents of Children with Disruptive Behavior Disorders. Hamilton, Ontario, Canada: COPE Works.

Cunningham, C. E., Davis, J. R., Bremner, R., Dunn, K. W., \& Rzasa, T. (1993). Coping modeling problem solving versus mastery 
modeling: Effects on adherence, in-session process, and skill acquisition in a residential parent-training program. Journal of Consulting and Clinical Psychology, 61, 871-877.

Dadds, M. R., \& McHugh, T. A. (1992). Social support and treatment outcome in behavioral family therapy for child conduct problems. Journal of Consulting and Clinical Psychology, 60, 252-259.

Deater-Deckard, K. (2004). Parenting stress. New Haven, CT: Yale University Press.

Dumas, J. E., \& Wahler, R. G. (1983). Predictors of treatment outcome in parent training: Mother insularity and socioeconomic disadvantage. Behavioral Assessment, 5, 301-313.

D'Zurilla, T. J., \& Nezu, A. M. (1999). Problem-Solving Therapy: A social competence approach to clinical intervention, (2nd ed.) New York: Springer.

Eyberg, S. M., Edwards, D., Boggs, S. R., \& Foote, R. (1998). Maintaining the treatment effects of parent training: The role of booster sessions and other maintenance strategies. Clinical Psychology: Science and Practice, 5(4), 544-554.

Fabiano, G. A., Pelham, W. E., Gnagy, E. M., Kipp, H., Lahey, B. B., Burrows-MacLean, L., et al. (2006). The reliability and validity of the Children's Impairment Rating Scale: A practical measure of impairment in children with ADHD. Journal of Clinical Child and Adolescent Psychology, 35, 369-385.

Haskett, M. E., Ahern, L. S., Ward, C. S., \& Allaire, J. C. (2006). Factor structure of the Parenting Stress Index-Short Form. Journal of Clinical Child and Adolescent Psychology, 35, 302-312.

Hoza, B., Gerdes, A. C., Mrug, S., Hinshaw, S. P., Buckowski, W. M., Gold, J. A., et al. (2005). Peer-assessed outcomes in the Multimodal Treatment Study of children with attention-deficit/ hyperactivity disorder. Journal of Clinical Child and Adolescent Psychology, 34, 74-86.

Kazdin, A. E., Holland, L., Crowley, M., \& Brenton, S. (1997). Barriers to treatment participation scale: Evaluation and validation in the context of child outpatient treatment. Journal of Child Psychology and Psychiatry, 38, 1051-1062.

Kazdin, A. E., \& Mazurick, J. L. (1994). Dropping out of child psychotherapy: Distinguishing early and late dropouts over the course of treatment. Journal of Consulting and Clinical Psychology, 62, 1069-1074.

Kazdin, A. E., Mazurick, J. L., \& Bass, D. (1993). Risk for attrition in treatment of antisocial children and families. Journal of Clinical Child Psychology, 22, 2-16.

Kazdin, A. E., \& Wassell, G. (2000). Predictors of barriers to treatment and therapeutic change in outpatient therapy for antisocial children and their families. Mental Health Services Research, 2, $27-40$.

Kazdin, A. E., \& Whitley, M. K. (2003). Treatment of parental stress to enhance therapeutic change among children referred for aggressive and antisocial behavior. Journal of Consulting and Clinical Psychology, 71, 504-515.

Lundahl, B., Risser, H. J., \& Lovejoy, C. (2006). A meta-analysis of parent training: Moderators and follow-up effects. Clinical Psychology Review, 26, 86-104.

McKay, M. M., Nudelman, R., McCadam, K., \& Gonzales, J. (1996). Evaluating a social work engagement approach to involving innercity children and their families in mental health care. Research on Social Work Practice, 6, 462-472.

McKay, M. M., Stoewe, J., McCadam, K., \& Gonzales, J. (1998). Increasing access to child mental health services for urban children and their caregivers. Health \& Social Work, 23, 9-15.

Miller, G. E., \& Prinz, R. J. (1990). Enhancement of social learning family interventions for childhood conduct disorder. Psychological Bulletin, 108, 291-307.

Morrissey-Kane, E., \& Prinz, R. J. (1999). Engagement in child and adolescent treatment: The role of parental cognitions and attributions. Clinical Child and Family Psychology Review, 2, 183-198.

Nock, M. K. (2003). Progress review of the psychosocial treatment of child conduct problems. Clinical Psychology: Science and Practice, $10,1-28$.

Nock, M. K., \& Kazdin, A. E. (2001). Parent expectancies for child therapy: Assessment and relation to participation in treatment. Journal of Child and Family Studies, 10, 155-180.

Nock, M. K., \& Kazdin, A. E. (2005). Randomized controlled trial of a brief intervention for increasing participation in parent management training. Journal of Consulting and Clinical Psychology, 73, 872-879.

Parenting Clinic. (2000). Dyadic parent-child interactive coding system: A manual. Seattle: University of Washington.

Pelham, W. E. (2002). Attention Deficit Hyperactivity Disorder: Diagnosis, assessment, nature, etiology, and treatment. Buffalo, NY: CTADD.

Pelham, W. E., \& Fabiano, G. A. (2008). Evidence-based psychosocial treatment for attention-deficit/hyperactivity disorder: An update. Journal of Clinical Child and Adolescent Psychology, 37, 184-214.

Pelham, W. E., Fabiano, G. A., \& Massetti, G. M. (2005). Evidencebased assessment of attention-deficit/hyperactivity disorder in children and adolescents. Journal of Clinical Child and Adolescent Psychology, 34, 449-476.

Pelham, W. E., Gnagy, E. M., Greenslade, K. E., \& Milich, R. (1992). Teacher ratings of $D S M-I I I-R$ symptoms for the disruptive behavior disorders. Journal of the American Academy of Child and Adolescent Psychiatry, 31, 210-218.

Pelham, W. E., Lang, A. R., Atkeson, B., Murphy, D. A., Gnagy, E. M., Greiner, A. R., et al. (1998). Effects of deviant child behavior on parental alcohol consumption: Stress-induced drinking in parents of ADHD children. American Journal of Addictions, 7, 103-114.

Pelham, W. E., Wheeler, T., \& Chronis, A. (1998). Empirically supported psychosocial treatments for ADHD. Journal of Clinical Child Psychology, 27, 190-205.

Pfiffner, L. J., Jouriles, E. N., Brown, M. M., Etscheidt, M. A., \& Kelly, J. A. (1990). Effects of problem solving therapy on outcomes of parent training for single parent families. Child and Family Behavior Therapy, 12, 1-11.

Prinz, R. J., \& Miller, G. E. (1994). Family-based treatment for childhood antisocial behavior: Experimental influences on dropout and engagement. Journal of Consulting and Clinical Psychology, 62, 645-650.

Prinz, R. J., \& Miller, G. E. (1996). Parental engagement in interventions for children at risk for conduct disorder. In R. Dev Peters \& R. J. McMahon (Eds.), Preventing childhood conduct disorders, substance abuse and delinquency (pp. 161-183.) Thousand Oaks, CA: Sage.

Renk, K., \& Phares, V. (2004). Cross-informant ratings of social competence in children and adolescents. Clinical Psychology Review, 24, 239-254.

Robinson, E. A., \& Eyberg, S. M. (1981). The dyadic parent-child interaction coding system: Standardization and validation. Journal of Consulting and Clinical Psychology, 49, 245-250.

Sanders, M. R., \& McFarland, M. (2000). Treatment of depressed mothers with disruptive children: A controlled evaluation of cognitive behavioral family intervention. Behavior Therapy, 31, 89-112.

Spaccarelli, S., Cotler, S., \& Penman, D. (1992). Problem-solving skills training as a supplement to behavioral parent training. Cognitive Therapy and Research, 16, 1-18.

Swanson, J. M., Kraemer, H. C., Hinshaw, S. P., Arnold, L. E., Conners, C. K., Abikoff, H. B., et al. (2001) Clinical relevance of the primary findings of the MTA: Success rates based on severity of ADHD and ODD symptoms at the end of treatment. Journal of the American Academy of Child \& Adolescent Psychiatry, 40, 168-179. 
Webster-Stratton, C. H. (1985). The effects of father involvement in parent training for conduct problem children. Journal of Child Psychology and Psychiatry, 26, 801-810.

Webster-Stratton, C. (1989). The relationship of marital support, conflict, and divorce to parent perceptions, behaviors, and childhood conduct problems. Journal of Marriage and the Family, 51, 417-430.
Webster-Stratton, C., \& Hammond, M. (1990). Predictors of treatment outcome in parent training for families with conduct problem children. Behavior Therapy, 21, 319-337.

Wells, K. C., Abikoff, H., Abramowitz, A., Courtney, M., Cousins, L., Del Carmen, R., et al. (1994). Parent training manual: MTA study. Unpublished manuscript. 
Copyright of Journal of Clinical Child \& Adolescent Psychology is the property of Lawrence Erlbaum Associates and its content may not be copied or emailed to multiple sites or posted to a listserv without the copyright holder's express written permission. However, users may print, download, or email articles for individual use. 